\title{
THE OXYGENATION OF CONCENTRATED VERSUS NORMAL BLOODS
}

\author{
By GEO. B. RAY, C. I. THOMAS AND J. E. STRONG 1 \\ (From the Department of Physiology, Western Reserve University, School of Medicine, \\ Cleveland)
}

(Received for publication June 28, 1933)

While the physiological activity of normal or dilute bloods represents one of the most widely studied fields of investigation, the problems presented by high concentrations of hemoglobin have not received the attention they merit. As far as the writers have been able to determine comparatively little attention has been paid to the ability of polycythemic bloods to take up oxygen under normal conditions. Barcroft and Murray (1) compared the effect of concentrating blood by centrifugating and removing part of the plasma. For a given $\mathrm{O}_{2}$ tension they found a higher degree of saturation in the concentrated blood than in normal blood which in turn took up more $\mathrm{O}_{2}$ than did a sample diluted with the plasma which had been taken from the concentrated blood. This variation was considered secondary to a disturbance in the carbondioxide equilibrium. Richards and Strauss (2) found no difference between the dissociation curve of the blood of a polycythemic patient and that of blood from a normal individual.

The experiments reported in this paper fall into two groups: the first designed to repeat the tests of the oxygen-combining power of concentrated blood in vitro, and the second to observe and compare the oxygenation of concentrated blood with that of normal blood in the perfused lung where the experimenter has a maximum control over the factors associated with oxygenation. Such a series of experiments should offer a concrete and definite answer to the question, does a plethora of red cells, per se, influence the respiratory function of the blood.

\section{Experiments on the $\mathrm{O}_{2}$-combining power of concentrated blood}

The technique employed in the handling of the blood and gases in these experiments was essentially that described by Austin et al. (3). The concentrated blood was obtained by centrifugating blood of normal dogs and pipetting away most of the supernatant plasma. A measured amount of this blood, of known oxygen capacity, was placed in a small tonometer bearing at one end a two-way stopcock and at the other a

1 Crile_Research Scholar. 
tube of fairly large bore. The tubular end was connected to a similar end of a large tonometer of the same type having a capacity of 250 to $300 \mathrm{cc}$. Care was taken to see that the blood filled the smaller tonometer well into the rubber connection. This connection was then clamped and the pair of tonometers connected with a manifold whereby one could either evacuate the vessel or supply $\mathrm{CO}_{2}$-free air, nitrogen, or carbon dioxide. The tonometer was repeatedly evacuated and washed with nitrogen after which sufficient $\mathrm{CO}_{2}$-free air was admitted to give the desired oxygen tension, following which the $\mathrm{CO}_{2}$ tension was adjusted to 40 millimeters. Pressure within the tonometer was then brought to atmospheric by the admission of nitrogen. With the cocks closed the tonometer was removed from the manifold and the clamp between the blood and gas chamber taken off, allowing the blood to flow into the larger chamber. In this manner portions of each sample of blood were brought into equilibrium with gas mixtures of five different oxygen tensions. The tonometers were rotated simultaneously in an air bath at $37^{\circ} \mathrm{C}$. for thirty minutes. At the end of the period of equilibration the blood was allowed to return to the small chamber and the clamp replaced. The chambers were then disconnected, and samples taken for analysis by forcing the blood into a pipette with mercury pressure. At no time was the blood allowed to come in contact with the room air. The analyses for oxygen content were made with the Van Slyke and Neill constant volume manometric apparatus; oxygen capacity was estimated by means of the spectrophotometer (Ray, Blair and Thomas (4)). The final $\mathrm{O}_{2}$ tensions existing in the tonometers were corrected for oxygen lost from the blood and for the effect of temperature change.

The results of a characteristic series of experiments are given in Figure 1. The open circles represent the results found in the normal blood and the black those for the polycythemic blood. Both samples were from the same original blood in order to avoid any chemical factors which might influence the results. These data show beyond any doubt that the oxygen-combining power of the two bloods is identical, under the conditions of the experiments. In fact the distribution of the two sets of points coincides quite as well as would points secured from two samples of the same concentration. The variation of any point from the mean curve is within the experimental error of the methods employed.

There is a point of distinction which can not be demonstrated in the graph, but was definitely apparent during the course of the experiment. The time required for the concentrated blood to reach an equilibrium was markedly longer than that needed for normal blood. While the normal sample showed its maximal color change in five to ten minutes after the start of equilibration the concentrated needed almost double the time for the same change. This was a natural sequence of the concentration of the blood since diffusion is inversely proportional to viscosity 


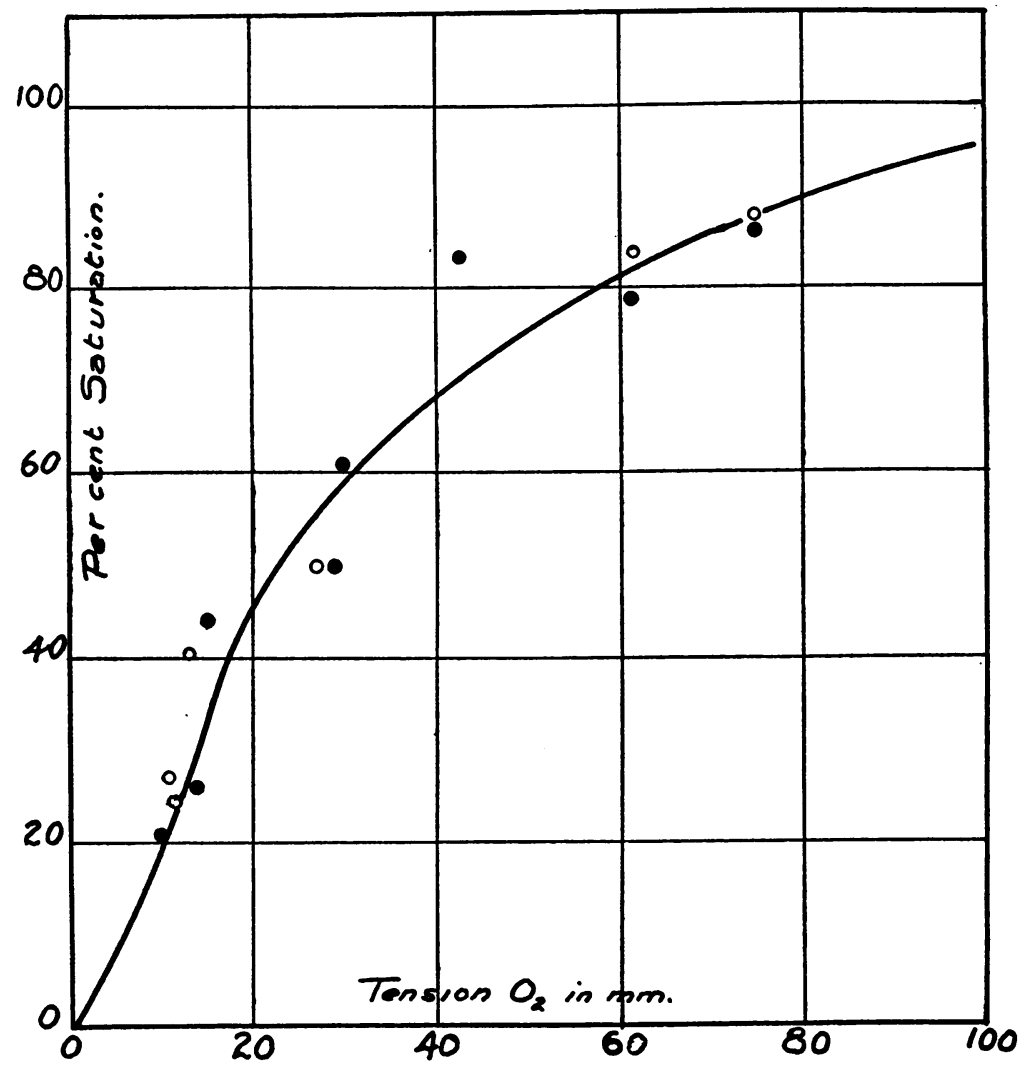

Fig. 1. Dissociation Curve of Normal and Polycythemic Bloods

The open circles represent blood of normal hemoglobin concentration and the black, polycythemic blood.

and also the amount of gas moved was greatly increased. The effect of both factors will be discussed in detail in a later section.

\section{Experiments with the perfused lung}

The preceding experiments give no tangible evidence of a difference between the oxygen combining power of the two types of blood. Obviously if a difference does exist it must be sought in other directions. We turned, therefore, to the perfused lung. These experiments were planned to determine if a normal or approximately normal oxygenating apparatus was capable of working with its usual efficiency when the number of cells passing through the tissue per unit of time was increased. That is, if the rate of flow of hemoglobin through the lung is doubled can the respiratory conditions be so adapted as to produce a normal degree of oxygenation? The importance of blood flow through a tissue 
has often been stressed, but in this case it is, perhaps, preferable that attention be directed to the amount of hemoglobin as the essential factor.

In order to study this effect we were forced to use a perfused lung. Preliminary experiments on intact anesthetized dogs gave uncertain results. The results reported in this section were made on the isolated lungs of cats with the conditions of ventilation, blood flow, etc., made to simulate as closely as possible, normal conditions, while allowing full control over every variable. It was thus possible to study the respiratory function without the interference of the generalized compensatory mechanisms which were likely to be brought into play in the intact animal in response to a sudden massive injection of corpuscular cream.

A diagram of the apparatus used in perfusing the lung is given in Figure 2. The lungs (1) and heart were removed as rapidly as possible from the anesthetized cat. In order to prevent clot formation during this process the animal was previously injected with heparin. A cannula (2) was inserted into the pulmonary artery by way of the right ventricle. The tracheal cannula (3) was tied in. The outflow cannula (4) was inserted into the left auricle by way of the ventricle and the whole preparation placed in the jar (5) which served as a thorax. The metal plate (6) was clamped in place on a vaselined rubber ring, making an air-tight seal. The basic negative pressure was controlled by connecting tube (8) to a vacuum and expanding the lungs to approximately their normal size. This pressure was controlled by a manometer. The fluctuating negative pressure was produced by a pump (7) having a variable stroke and speed. A tambour connected at $(9)$ recorded these fluctuations of pressure and so, upon calibration, served as a measure of the tidal air volume. This estimation was checked by a water manometer connected at $(10)$ to the tracheal tube. The main branch of the tracheal tube went to a pair of mercury valves (11) of the type described by Bailey (5), which allowed passage of air with a minimum of resistance.

For ventilating the lung atmospheric air enriched with approximately 5 per cent carbon dioxide was used, in order to maintain the acid-base relationships of the blood. This was stored in a 100 liter spirometer connected at (12) from which it was drawn into a smaller graduated spirometer (13) for use. The composition of the stored gas was checked from time to time to detect changes in $\mathrm{O}_{2}$ and $\mathrm{CO}_{2}$ content.

The blood used in these experiments was citrated ox blood. It is unfortunate that blood of another species had to be used, but the difficulties associated with collecting a sufficient amount of fresh cats' blood were insurmountable. Careful preliminary experiments were carried out to detect any indication of harmful reactions resulting from the above combination. Even after long periods of perfusion no deleterious effect could be seen other than the appearance of some edema which might have been expected, regardless of the nature of the perfusion fluid. 


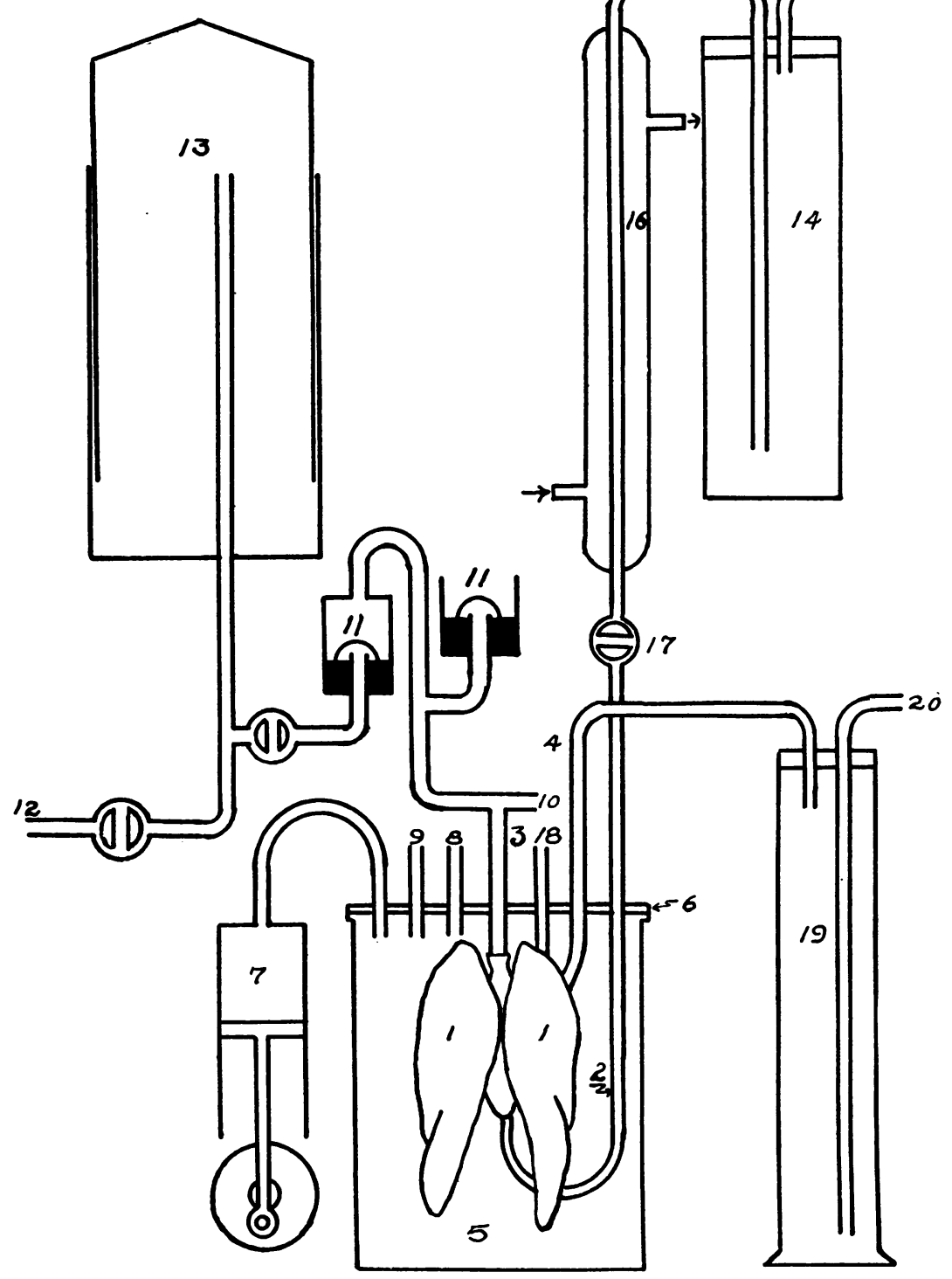

Fig. 2. Apparatus Used in Perfusing the Lung Explanation of the figure is given in the text. 
The fact that continued perfusions caused no striking changes in blood flow or ventilation was deemed evidence of the nontoxicity of the blood.

The blood to be used in any particular series of experiments was divided in advance into two portions. One, the larger, was centrifugated and most of the supernatant plasma removed. Just previous to the experiment the sample to be used at that time was placed in a large bottle tightly stoppered with a two-hole rubber stopper, one opening of which was connected to a manifold by means of which vacuum, nitrogen and carbon dioxide could be controlled. The other opening of the bottle was tightly closed. The bottle was then evacuated and the resulting foam broken by the admission of nitrogen. This process was repeated until a vacuum produced no foam, which was taken as an indication that reduction was practically complete. In the case of concentrated bloods the process was, of course, much slower, but was accelerated by saturating the blood with carbon dioxide. The experiments might be criticized as unphysiological since blood of such low $\mathrm{O}_{2}$ content was used, but it must be remembered that it was desired to test the ability of the lung to allow access of oxygen to the blood. Unless some common base line were adopted this estimation could not be made under exactly comparable conditions. The reduced bloods were kept under an atmosphere of nitrogen until they were ready to be introduced into the perfusion apparatus. At this time the sample was forced into the storage bottle of the apparatus (14) by pressure from a hydrogen tank and was kept under an atmosphere of this gas during the course of the experiment. The storage bottle was connected to an 18 liter bottle connected at (15), which in turn was connected to a constant water pressure. The blood was forced from the storage bottle through a condenser (16) to bring it to the proper temperature, and thence to the pulmonary artery. The pressure in this artery was adjusted by means of the stopcock (17). A record of the pulmonary arterial pressure changes was recorded by a mercury manometer connected at (18). The outflowing blood was caught in a $500 \mathrm{cc}$. graduate (19) which served to measure the total blood flow while the rate of flow was computed from a tambour connected at (20).

Perhaps the feature to be most carefully avoided was the possibility of edema. In the preliminary experiments in which perfusion was carried out over a long period of time, edema was obviously present. In the course of these experiments certain signs were discovered whereby it was possible to recognize this condition at its onset and furthermore to estimate the duration of time that an experiment could be carried on without vitiating the results. Early edema was manifested by an increase in ventilation pressure and a decrease in venous pressure. No experiments in which these signs appeared are-reported in this paper. In all experiments the period of perfusion was made as brief as was consistent with securing an adequate series of samples. 
The analyses for the degree of saturation were made on the Van Slyke apparatus, while the oxygen capacity was estimated from the hemoglobin concentration determined by means of the spectrophotometer. Both arterial and venous samples were taken as nearly simultaneously as possible for each reading. Any variation in hemoglobin content served as a check on fluid loss, and hence edema. Obviously care had to be exerted that the storage bottle of blood was constantly agitated in order to avoid sedimentation. In the first experiments we attempted to study both normal and polycythemic bloods in the same lung, but the period of perfusion extended over much too long a time and the practice had to be discontinued in favor of individual experiments for each type of blood.

The general nature of the data obtained by this procedure may be illustrated in tabular form by a protocol of a pair of companion experiments.

It will be noted that the degree of saturation of the venous blood in the case of the concentrated sample dropped markedly in the later

TABLE I

Protocol of a typical pair of experiments

\begin{tabular}{|c|c|c|c|c|c|c|c|c|c|c|c|}
\hline \multirow[b]{2}{*}{$\begin{array}{c}\text { Experi- } \\
\text { ment }\end{array}$} & \multirow[b]{2}{*}{ Time } & \multicolumn{2}{|c|}{$\begin{array}{c}\text { Blood } \\
\text { pressure }\end{array}$} & \multicolumn{2}{|c|}{$\begin{array}{c}\text { Intrathoracic } \\
\text { pressure }\end{array}$} & \multicolumn{3}{|c|}{ Arterial blood } & \multicolumn{3}{|c|}{ Venous blood } \\
\hline & & $\begin{array}{c}\text { Arte- } \\
\text { rial }\end{array}$ & Venous & $\begin{array}{l}\text { Inspi- } \\
\text { ration }\end{array}$ & $\begin{array}{l}\text { Expi- } \\
\text { ration }\end{array}$ & $\begin{array}{c}\text { Oxygen } \\
\text { capacity }\end{array}$ & $\begin{array}{l}\text { Oxygen } \\
\text { content }\end{array}$ & $\begin{array}{c}\text { Per } \\
\text { cent } \\
\text { satu- } \\
\text { ration }\end{array}$ & \begin{tabular}{|} 
Oxygen \\
capacity
\end{tabular} & $\begin{array}{l}\text { Oxygen } \\
\text { content }\end{array}$ & $\begin{array}{l}\text { Per } \\
\text { cent } \\
\text { satu- } \\
\text { ration }\end{array}$ \\
\hline \multirow{5}{*}{$A^{*}$} & minutes & $\underset{H \boldsymbol{g}}{m m}$ & $\underset{\mathrm{H}}{\mathrm{mm}} \mathrm{O}$ & ${ }_{\mathrm{H}_{2} \mathrm{O}}^{\mathrm{O}}$ & ${ }_{H_{2} \mathrm{O}}$ & $\begin{array}{l}\text { volumes } \\
\text { per cent }\end{array}$ & $\begin{array}{l}\text { volumes } \\
\text { per cent }\end{array}$ & & $\begin{array}{l}\text { volumes } \\
\text { per cent }\end{array}$ & $\begin{array}{l}\text { volumes } \\
\text { per cent }\end{array}$ & \\
\hline & 1.25 & 10 & 10 & -50 & -20 & 15.34 & 4.32 & 28 & 14.74 & 14.83 & 100.0 \\
\hline & 3.60 & 20 & 25 & -40 & -10 & 15.27 & 4.08 & 26.8 & 15.14 & 15.13 & 100.0 \\
\hline & 4.80 & 10 & 25 & -100 & -50 & 15.42 & 4.18 & 27.25 & 15.42 & 14.83 & 96.0 \\
\hline & 7.50 & 40 & 45 & -60 & -30 & lost & 4.08 & & 15.42 & 14.90 & 96.5 \\
\hline \multirow[t]{3}{*}{ Bt } & 1.00 & 23 & 24 & -80 & -40 & 39.25 & 1.42 & 3.60 & 37.59 & 26.82 & 71.4 \\
\hline & 3.20 & 23 & 24 & -110 & -40 & 40.59 & 1.42 & 3.50 & 37.37 & 24.58 & 66.0 \\
\hline & 6.50 & 23 & 24 & -110 & -40 & 39.39 & 1.42 & 3.60 & 38.83 & 21.88 & 56.3 \\
\hline
\end{tabular}

* Experiment A. Tidal air, 28 cc. per respiration. Rate of respiration, 40 per minute. Lung capacity, $196 \mathrm{cc}$. Average rate of blood flow, $35 \mathrm{cc}$. per minute.

† Experiment B. Tidal air, $30 \mathrm{cc}$. per respiration. Rate of respiration. 20 per minute. Lung capacity, $166 \mathrm{cc}$. Average rate of blood flow, $40 \mathrm{cc}$, per minute. 
readings. As far as we could determine this was not due to any change in the lung tissue but rather to a continually diminishing tension of oxygen in the alveolar air resulting from the demands of the concentrated blood. Spectrophotometric evidence proved, in addition, that the hemoglobin had retained its ability to combine with oxygen. The slight variations noted in oxygen capacity are without doubt due to sedimentation, which could not be completely controlled.

The results are summarized in Figure 3 in which the crosses represent the degree of saturation of the normal blood and the circles that of the concentrated blood. It will be noted that in every case the normal blood was oxygenated completely or almost completely, which is exactly what one would predict for the oxygen tensions used and the rate at which the blood was flowing through the lungs. The results indicate clearly the efficiency of the perfusion system.

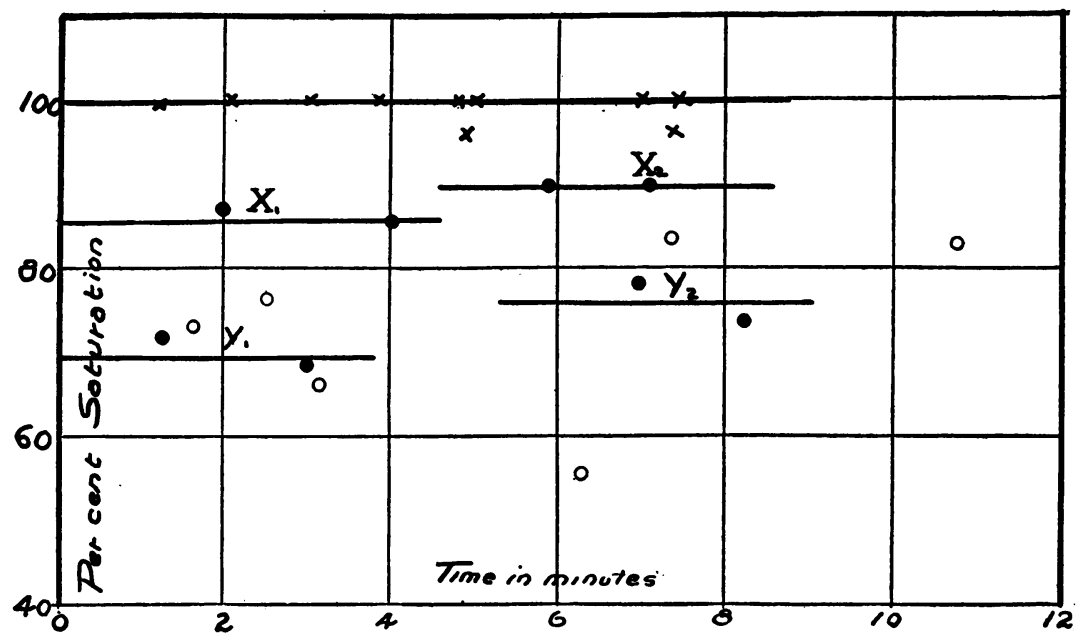

Fig. 3. Comparative Results of Perfusing the Lungs with Normal AND Polycythemic Blood

Crosses represent normal blood; the circles, polycythemic. Explanations of curves $X_{1}, X_{2}, Y_{1}$ and $Y_{2}$ are given in the text.

In the concentrated blood on the other hand, a normal saturation was never attained. In spite of the fact that the lungs were to all intents and purposes normal, i.e. allowed oxygenation of normal blood, it is evident that with the usual ventilation and blood flow the system is incapable of functioning adequately. The incapacity of the system may be noted in a very simple manner. In two experiments, represented by curves $X$ and $Y$, an attempt was made to reproduce what would be the normal compensatory reaction of an animal, i.e. the minute volume of respiration was increased. These results are of particular significance 
since they show beyond question that the difficulty is not referable to any faults of the apparatus. In the routine experiments ventilation was within the normal resting limits defined by Scott (6) and by Churchill (7), whose observations agreed with our own measurements. It was further noted that hyperpnea, produced by stimulation of the sciatic previous to removing the heart and lung, raised the ventilation to about twice its resting value. Accordingly the minute volume was doubled in order to test the effect of a normal degree of hyperpnea.

The ventilation in the section of the first curve, designated as $X_{1}$, was $700 \mathrm{cc}$. per minute, which is within the normal range. At point $X_{2}$ the ventilation was increased to $1330 \mathrm{cc}$. per minute. The increase in saturation is, however, far from proportional to the increase in ventilation, since the change is only from 87 to 90 per cent, which would correspond to a change in alveolar $\mathrm{O}_{2}$ tension of about 5 millimeters.

The results plotted in curve $Y$ are more striking. The original ventilation, represented by section $Y_{1}$ of the curve, was $1200 \mathrm{cc}$. per minute while in the section $Y_{2}$ the volume was increased to $2280 \mathrm{cc}$. per minute. In this case we have started with a moderate hyperpnea and increased the ventilation 190 per cent. In spite of this oxygen increase the saturation of the blood changes only from 70 to 77 per cent, equivalent, approximately, to $9 \mathrm{~mm}$. of $\mathrm{O}_{2}$. It is obvious that if this represents the proportionality between ventilation and saturation, a tremendous hyperpnea would be required to produce a normal condition in the venous blood. It is conceivable that the oxygen saturation of the blood failed to increase under the unphysiological artificial conditions of these experiments because forced expiration did not have its usual effect, that no matter what the minute volume might be, the alveoli were incompletely aerated and therefore contained air with an unusually low oxygen tension. In order to test the validity of such a criticism the mode of attack was changed. Instead of increasing the amount of air sucked into the lungs at each respiration the amount of oxygen in the air was increased. Under these circumstances the problem of accumulation of nitrogen in the alveoli was eliminated and oxygen saturation depended only upon the rate of diffusion into the blood and subsequent oxygenation. In accordance with this scheme the ventilation gas mixture was changed to 94 per cent oxygen and 6 per cent carbon dioxide. The results proved definitely that even such a concentration of oxygen was barely adequate to approximate normal conditions; with this tension the average saturation was only 91 per cent. This figure indicates that it is about 6 to 7 times as difficult to oxygenate concentrated blood as it is to oxygenate normal blood.

\section{DISCUSSION}

Certain definite facts stand out as the result of the above experiments. $A$. With this method blood of normal concentration is adequately 
oxygenated. $B$. Increasing the number of cells, and consequently the amount of hemoglobin passing through the lung tissue per minute, results in inadequate oxygenation. $C$. Doubling the amount of ventilation results in only a minor change in the saturation of concentrated blood. $D$. Only when the oxygen tension of the inspired gas is increased to over six times that of the normal atmosphere does the saturation approximate normal.

The question arises as to what physiological reactions are responsible for these differences between bloods of normal and high concentration. The distinction appears to be inherent in the character of the blood and not referable to the conditions of the experiment, since normal blood behaves as it does when it is oxygenated in the physiological manner. The lungs function efficiently as an oxygenating device. In fact, as the blood used was nearly completely reduced the tissue was called upon to do more work than would ordinarily be the case in vivo. The use of blood from another species seems to have caused no toxic effects. On the basis of these observations we are inclined, therefore, to dismiss the idea that there was any change in the lung tissue per se.

The problem therefore resolves itself into a discussion of what factors in the blood or respiratory mechanism act, either independently or together, to produce the result found. The outstanding factor is perhaps the increased amount of hemoglobin passing through the lungs per minute. The demand for oxygen increases directly as the concentration of hemoglobin. In consequence, oxygen tension in the alveoli and the degree of oxygenation of the blood should diminish as the perfusion progresses. That this is the case is illustrated by the experiment shown in Experiment B, Table I, where the saturation decreased from 71 to 56 per cent during the period of measurement.

This might afford an adequate explanation of the whole phenomenon, were it not for the fact that increased ventilation of the lungs proved insufficient to produce normal oxygenation and also that it was found necessary to increase the oxygen tension to seven times that of normal before full saturation was even approximated. In the case of the experiments in which the ventilation was increased, the change in the degree of saturation was by no means proportional to the increase in the minute volume of respiration. The increased movement of air failed to change the tension of alveolar oxygen to any marked extent. Perhaps more significant is the high pressure necessary to drive a sufficient amount of oxygen into the blood. In other words, the rate of diffusion of oxygen is reduced in the case of the more concentrated blood. This is exactly what Harrop and Heath (8) found in polycythemic individuals. These investigators ascribe this change to alteration of the pulmonary epithelium. This cannot be said to be true in our experiments, inasmuch as the lungs behaved in a normal manner when perfused with blood 
containing a low concentration of cells. It is obvious, therefore, that the reason for the faulty oxygenation of the blood must be sought elsewhere. It will be recalled that in the experiments upon the dissociation curves a longer time was needed for complete equilibration of the concentrated blood. It would seem, therefore, that a certain fraction of the unsaturation can be attributed to the character of the concentrated blood itself.

Still another factor to be considered is the reaction which occurs in the capillaries. In the table cited it will be noted that the amount of blood flowing through the system is approximately the same in both experiments. The viscosity of the concentrated blood is much greater than normal, yet if the second readings of both types of experiments are compared it will be noted that the same pressure produces the same flow. The simplest explanation that can be suggested for this adjustment is dilatation of the smaller vessels. The appearance of the lungs in the two cases certainly substantiated this view. If such a compensation involved the alveolar capillaries a second reason for the impairment of oxygen diffusion is found in the increased distance the gas must pass in order to complete the process of oxygenation. In addition to this effect, the engorgement of the vessels would decrease the alveolar space, i.e., the vital capacity, thereby adding to the factors which promote anoxemia.

\section{SUMMARY}

Studies of the dissociation curves of normal and artificial polycythemic bloods, show no difference in the tension of oxygen required to produce a given saturation. When these types of blood are oxygenated by the perfused lung the normal blood becomes completely oxygenated while the concentrated blood is never fully saturated. Increasing the oxygen tension increases the saturation of the concentrated blood.

The difference between the two bloods is ascribed to the greater rate at which hemoglobin in the polycythemic blood passes through the lungs, coupled with a delayed diffusion resulting from capillary dilatation.

\section{BIBLIOGRAPHY}

1. Barcroft, J., and Murray, C. D., Phil. Trans. Roy. Soc., (B), 1923, ccxi, 469. Some Secondary Effects of Increasing the Proportion of Red Corpuscles in Blood.

2. Richards, D. W., Jr., and Strauss, M. L., J. Clin. Invest., 1927, iv, 105. Oxyhemoglobin Dissociation Curves of Whole Blood in Anemia.

3. Austin, J., Cullen, G. E., Hastings, A. B., McLean, F. C., Peters, J. P., and Van Slyke, D. D., J. Biol. Chem., 1922, liv, 121. Studies of Gas and Electrolyte Equilibria in Blood. I. Technique for Collection and Analysis of Blood and for its Saturation with Gas Mixtures of Known Composition.

4. Ray, G. B., Blair, H. A., and Thomas, C. I., J. Biol. Chem., 1932, xcviii, 63. The Spectrophotometric Determination of Certain Blood Pigments. 
5. Bailey, C. V., Proc. Soc. Exper. Biol. and Med., 1926, xxiv, 184. A Low Resistance Air Valve.

6. Scott, R. W., Am. J. Physiol., 1917, xliv, 196. The Effect of the Accumulation of Carbon Dioxide on the Tidal Air and on the $\mathrm{H}$-ion Concentration of the Arterial Blood in the Decerebrate Cat.

7. Churchill, E. D., Am. J. Physiol., 1928, lxxxvi, 274. The Effect of Increased Blood Flow on the Ratio between Oxygen Consumption and Pulmonary Ventilation.

8. Harrop, G. A., Jr., and Heath, E. H., J. Clin. Invest., 1927, iv, 53. Pulmonary Gas Diffusion in Polycythemia Vera. 